\title{
Nobel laureates are not hot
}

\author{
Marek Kosmulski ${ }^{1}$
}

Received: 8 November 2019 / Published online: 22 February 2020

(c) The Author(s) 2020

\begin{abstract}
The achievements of all 97 Nobel laureates in Chemistry, Economy, Medicine, and Physics of the period 2010-2019 are compared with achievements of top non-Nobel scientists in terms of standard bibliometric indicators (number of publications, number of citations, Hirsch index), of the numbers of highly cited papers and of hot papers (as defined by WoS $®$ ), and of $c$, a composite score (Ioannidis et al. in PLoS Biol 14:e1002501, 2016). Ninety recent Nobel laureates were in top 100,000 scientists in terms of $c$, and 45 recent Nobel laureates were in top 6000 scientists in terms of $c$. Only 32 recent Nobel laureates were in top 6000 scientists in terms of ch (Hirsch-type index with self-citations excluded), 32 recent Nobel laureates were in top 6000 scientists in terms of the number of citations (self-citations excluded), 17 recent Nobel laureates were among the 6000 Highly Cited Researchers (WoS®), 4 recent Nobel laureates were in top 6000 scientists in terms of the number of hot papers, and 2 recent Nobel laureates were in top 6000 scientists in terms of the number of highly cited papers.
\end{abstract}

Keywords Nobel laureate $\cdot$ Citations $\cdot$ H-index

\section{Introduction}

There is a general agreement in the literature, that the achievements of Nobel laureates in terms of various bibliometric indicators are superior to the achievements of average scientists, especially in terms of the number of citations and of bibliometric indicators derived from the citation records. For example Garfield (1986) emphasized that most Nobel laureates of that time had extraordinary ability to publish highly-cited papers. Eighty-three percentage of the Nobel laureates had written at least one paper each with more than 300 citations (which is a very high number even by today's standards). Various aspects of publication and citation records of Nobel laureates have been discussed in the literature, and the number of papers, in which their bibliometric data were analyzed is very high. Yet a vast majority of those publications were focused on specific narrow aspects of the bibliometric records of Nobel laureates, e.g., they only considered a few specific papers authored by Nobel laureates (Sangwal 2015), a few Nobel laureates (Kosmulski 2012), Nobel laureates

Marek Kosmulski

m.kosmulski@pollub.pl

1 Lublin University of Technology, Lublin, Poland 
from one discipline (Boettke et al. 2012), did not compare Nobel laureates with top nonNobel scientists (Kreiner 2016), etc. In contrast we can hardly find comprehensive studies involving many Nobel laureates, many different bibliometric indicators, results from various databases, and comparison with top non-Nobel scientists.

The publication and citation habits of scientists are changing over time, and the relationships reported in older literature are not necessarily valid today. For example Biglu (2008) found that the mean number of references per paper increased by a factor of 4 between 1970 and 2005. The systematic increase in the mean value of references per paper, authors per paper, papers per journal, and in the numbers of journals covered by citation databases result in substantial increase in the number of citations of all scientists, even those who do not publish any more. Moreover different databases cover various journals and different time periods, and the results of studies based on different databases are not compatible. Table 1 shows the $h$ index of top- $h$ scientists in biomedical research identified by Hirsch (2005), including one Nobel laureate, taken from the original paper by Hirsch (2005) and their contemporary $h$ indices taken from various sources.

The $h$ index based on WoS increased in all five scientists, but to different degree (number five in 2006 became number one in 2019). The contemporary $h$ index based on WoS is higher than that based on Scopus in all five scientists. This is because they published their important papers before 1995, and a substantial fraction of their citations received before 1995 is neglected in Scopus. Finally only two scientists have their profiles in Google Scholar, which produced substantially higher $h$ than $h$ based on the other databases. Table 1 shows substantial discrepancies, which can result in different and even in contradictory conclusions.

Ioannidis et al. (2016) analyzed the achievements of 47 Nobel laureates of 2011-2015 and of top 30,000 scientists in terms of six bibliometric indicators based on their citation records for the year 2013. These indicators are: number of citations, Hirsch (2005) $h$ index, Schreiber (2009) hm index, number of citations to single-authored papers, number of citations to first-authored papers, and number of citations to first-or-lastauthored papers, all extracted from Scopus. Certainly the single author is also the first and the last author. The hm index is a co-authorship-adjusted Hirsch index which favors the authors who published their highly-cited papers alone or with small number of coauthors (Schreiber 2009). This should be emphasized that these indices are based on citations received in 2013, and their values are lower by an order of magnitude than analogous values calculated for the same scientists taking into account their life-time achievements. On top of the above indices Ioannidis et al. (2016) introduced a composite score $c$, which is a sum of six aforementioned indicators (standardized and logtransformed). Although the indicators were taken with equal weights, $c$ showed a high

Table 1 Hirsch index of selected scientists from different sources

\begin{tabular}{|c|c|c|c|c|c|}
\hline Name & Nobel & $\begin{array}{l}\text { Hirsch (2005), } \\
\text { WoS }\end{array}$ & WoS (2019) & Scopus (2019) & $\begin{array}{l}\text { Google } \\
\text { Scholar } \\
(2019)\end{array}$ \\
\hline SH Snyder & & 191 & 227 & 214 & 275 \\
\hline D Baltimore & 1975 & 160 & 196 & 175 & \\
\hline RC Gallo & & 154 & 163 & 139 & \\
\hline P Chambon & & 153 & 200 & 193 & \\
\hline B Vogelstein & & 151 & 232 & 230 & 263 \\
\hline
\end{tabular}


correlation with hm ( $r=0.92$ in top 84,116 scientists), and moderate correlation with $h$ $(0.25)$ and with the number of citations (0.11). Thirty-one Nobel laureates were in top 14,150 scientists in terms of $c$, but only 15 Nobel laureates were in top 14,150 in terms of the number of citations, and 18 Nobel laureates were in top 14,150 in terms of $h$.

Recently Ioannidis et al. (2019) presented an extended and updated version of their previous compilation (Ioannidis et al. 2016). On top of updated single-year results (year 2017 rather than 2013) they also presented lifetime achievements of top 100,000 scientists including multiple Nobel laureates in two versions (till 2017 and till 2018). Most results discussed in this study were taken from the most up-to-date version of lifetime achievements (Table S4 in Ioannidis et al. 2019), but analogous calculations were carried out with the older version (Table S1 in Ioannidis, et al. 2019), and the results were very similar. On top of the aforementioned six indicators and composite score, Ioannidis et al. (2019) report also the values of analogous six indicators with self-citations excluded, composite score with self-citations excluded, and other bibliometric indicators characterizing the top 100,000 scientists. To the best knowledge of the present author, the compilation by Ioannidis et al. (2019) is the most comprehensive compilation of scientific achievements of the most-cited scientists ever published, and it will be used for multisided comparison of Nobel laureates with non-Nobel scientists in the present study. Ioannidis et al. analyzed the achievements of Nobel laureates in their paper from 2016, but not in their recent paper (2019).

The ability of publishing highly cited and hot papers is an aspect of the scientific achievements of Nobel laureates, which has not received much attention in the bibliometric literature. Highly cited papers (papers published over the recent decade, which received enough citations to place them in the top $1 \%$ in certain field for certain publication year) and hot papers can be easily extracted from the WoS database. The current definition of hot papers (copied from WoS on December 27, 2019) is: "Hot Papers were published in the past 2 years and received enough citations in July/August 2019 to place them in the top $0.1 \%$ of papers in its academic fields". Since hot papers are limited to the recent 2 years, and highly cited papers are limited to the recent 10 years, the above assessment can only be used in active scientists or in scientists who quit their careers less than 10 years ago. The numbers of highly cited papers and especially of hot papers used as indicators of scientific achievements of an individual are very unstable in time (Kosmulski 2018). The list of highly cited papers is updated by WoS® every 2 months, and certain paper can appear, disappear and re-appear in the list. Unlike the number of citations and Hirsch index, which can only increase in time, the numbers of highly cited papers and especially of hot papers can increase, decrease or oscillate. Thus the numbers presented in this paper are only valid as of certain date.

The number of citations, Hirsch index, hm, and thus also the composite score $c$ are not suitable for comparison of achievements of scientists from different disciplines, and they put the scientists from disciplines with many scientists, many publications, and many references per paper (as Medicine) at inherent advantage. In contrast the number of highly cited and of hot papers as bibliometric indicators reflecting achievements of single scientist are field-normalized because of different thresholds in different disciplines. Field-normalization may play an important role when the achievements of economists are compared with chemists, physicists or medical doctors. On the other hand the numbers of papers, and citation rates in Chemistry, Medicine, and Physics are comparable, so field-normalization is not that crucial when only these 3 disciplines are compared. 


\section{Methods}

Nobel laureates in Chemistry, Economy, Medicine, and Physics awarded between 2010 and 2019 have been considered, and they will be referred to as recent Nobel laureates. These scientists were born between 1922 and 1974. Ninety-one of the total number of 97 recent Nobel laureates are still alive and at least 79 of them published scientific papers in 2017 or later. Their scientific achievements were recorded using different sources in October 2019, and compared with the achievements of non-Nobel scientists based on the same sources. Actually many Nobel laureates awarded before 2010 are still active and a few of them have extraordinary achievements. For example Alan J. Heeger (Nobel in Chemistry, 2000) is ranked 60 in citations, and 72 in $h$ according to Ioannidis et al. (2019). Yet the fraction of Nobel laureates awarded before 2010 among the scientists who are not recent Nobel laureates is very low.

The presence of recent Nobel laureates in Highly Cited Researchers was examined directly at https://hcr.clarivate.com/, and a special attention was paid to homonymous authors.

The numbers of hot and highly cited papers were extracted from the WoS $®$ core collection. The papers authored by recent Nobel laureates were examined one by one considering the topics of publications and the affiliations of the authors in order to avoid publications of homonymous authors, who are not Nobel laureates.

Records representing 90 recent Nobel laureates were found in Table S4 in Ioannidis et al. (2019) and they were used without corrections. These records were carefully selected among the records of homonymous authors (if applicable). The other 7 recent Nobel laureates were outside the 100,000 top-cited scientists according to the criteria used by Ioannidis et al. (2019). One recent Nobel laureate was found in Table S1 in Ioannidis et al. (2019), ranked 99,277 in the composite score, but not in more up-to-date Table S4. Two recent Nobel laureates were not found in Tables S1 or S4 in Ioannidis et al. (2019) in spite of their high $h$-indices and numbers of citations, which place them in top 6000 scientists. The other four recent Nobel laureates had relatively low $h$-indices and numbers of citations as compared with the top 100,000 scientists, so their absence in the compilation by Ioannidis et al. (2019) is not surprising.

\section{Results and discussion}

Seventeen recent Nobel laureates were among the 6000 Highly Cited Researchers HCR (WoS $®$ ) including 5 laureates in Chemistry, 3 in Economy, 7 in Medicine, and 2 in Physics. Nobel laureates in Economy were distinguished as HCR in Economy. The Nobel laureates in Physics were distinguished as HCR both in Physics and in materials science. Five Nobel laureates in Medicine were distinguished as HCR in different branches of Medicine, and two other in cross-field research. Only one Nobel laureate in Chemistry was distinguished as HCR in Chemistry, and the others were distinguished in biology, pharmacology, and in cross-field research. In other words all HCR in Chemistry but one are not recent Nobel laureates in Chemistry. Eighty (82\%) recent Nobel laureates were not among HCR. As the number of HCR (6000) was arbitrarily selected by WoS®, we will also examine the number of recent Nobel laureates in top 6000 scientists according to other criteria used in this study. 
Table 2 Hot papers by recent Nobel laureates by discipline

\begin{tabular}{llllc}
\hline & Chemistry & Economy & Medicine & Physics \\
\hline Hot papers & 8 & 1 & 5 & 11 \\
Authors of hot papers & 8 & 1 & 3 & 5 \\
HCR & 5 & 3 & 7 & 2 \\
HCR, authors of hot papers & 4 & 0 & 1 & 1 \\
\hline
\end{tabular}

Table 3 Hot papers by recent Nobel laureates and by other scientists

\begin{tabular}{lll}
\hline & \# of hot papers & Rank in hot papers \\
\hline Non-Nobel scientist & 38 & 1 \\
Two Nobel laureates in Physics & 8 each & $537-1422$ \\
One Nobel laureate in Medicine & 3 & $2981-4229$ \\
One Nobel laureate in Physics & 2 & $4230-8723$ \\
13 Nobel laureates & 1 each & $8724-46113$ \\
80 Nobel laureates & 0 each & $>46113$ \\
\hline
\end{tabular}

Seventeen recent Nobel laureates published together 25 hot papers out of the total number of 3238 hot papers of 46,113 authors. Hot papers had on average 22 authors each, one hot paper had 3388 authors, and 16 hot papers had over 1000 authors each. The multiauthorship in hot and in highly cited papers has been widely discussed in the literature (Noorhidawati et al. 2017; Kosmulski 2018; Dorta-González and Santana-Jiménez 2019). The achievements of recent Nobel laureates in hot papers are summarized in Table 2. 8 hot papers in physics were co-authored by 2 or 3 Nobel laureates. The concurrence between being HCR and ability to produce hot papers is observed in chemistry (4 out of 5 HRC have hot papers), but not in other disciplines (only 2 out of 12 HRC have hot papers). A rough estimate of the distribution of hot papers among their authors and the ranks of recent Nobel laureates by the number of hot papers are presented in Table 3. Apparently one author, who is not a recent Nobel laureate wrote as many as 38 hot papers. Table 3 properly reflects the order of magnitude of the numbers of scientists having at least one, at least two, etc. hot papers, and these numbers are substantially higher than the number of recent Nobel laureates. In other words, being an author of a hot paper may be considered as a great achievement at a level of an "ordinary scientist", but such an achievement is not comparable with Nobel Prize.

The ranks of recent Nobel laureates in Table 3 were not corrected for homonymous scientists. Most names in top 20 authors of hot papers are very common (e.g., Wang, Y), and they represent several homonymous scientists with different affiliations, and various scientific disciplines. Yet later in the list (ranks > 20) many names are not that popular, and this is very likely that they either represent single scientist each or they represent very few scientists each, of whom at least one has a substantial number of hot papers. The numbers representing ranks of two recent Nobel laureates having 8 hot papers each are probably overestimated, but the numbers representing ranks of the other recent Nobel laureates can very well be underestimated (there are certainly much more than 46,113 actual scientists who published at least one hot paper, because several apparent authors of multiple hot papers are in fact multiple homonymous authors). 
Table 4 Highly cited papers by recent Nobel laureates by discipline

\begin{tabular}{lllll}
\hline & Chemistry & Economy & Medicine & Physics \\
\hline Authors of highly cited papers & 19 & 8 & 17 & 17 \\
$\begin{array}{l}\text { Number of highly cited papers by the } \\
\text { top author }\end{array}$ & 37 & 8 & 39 & 59 \\
\hline
\end{tabular}

Table 3 suggests that the ability of publishing hot papers is not common among recent Nobel laureates, even if we consider that the deceased Nobel laureates could not have technically published any hot papers (hot papers are no more than 2 years old by definition). Eighty recent Nobel laureates have not published any hot papers, and this result is rather surprising considering a high number of scientists who have at least one hot paper as compared with the number of HCR.

Sixty-one recent Nobel laureates published highly cited papers. The total number of highly cited papers is 153,453 . The achievements of recent Nobel laureates in highly cited papers are summarized in Table 4.

All HCR among recent Nobel laureates had highly cited papers (3-59 papers). All recent Nobel laureates who had published hot papers had also published highly cited papers (1-58 papers). A rough estimate of the distribution of highly cited papers among their authors and the ranks of recent Nobel laureates by the number of highly cited papers are presented in Table 5 .

The problems with homonymous authors discussed above for hot papers are even more pronounced with highly cited papers, because there are 50 times more highly cited papers than the number of hot papers. Although the numbers in Table 5 are influenced by homonimity of the authors, they properly reflect the order of magnitude of the number of

Table 5 Highly cited papers by recent Nobel laureates and by other scientists

\begin{tabular}{lcl}
\hline & Highly cited papers & Rank in highly cited papers \\
\hline Non-Nobel scientist & 1089 & 1 \\
One Nobel laureate in Physics & 59 & $5012-5102$ \\
One Nobel laureate in Physics & 58 & $5103-5202$ \\
One Nobel laureate in Physics & 43 & $6552-6673$ \\
One Nobel laureate in Medicine & 39 & $7141-7352$ \\
One Nobel laureate in Chemistry & 37 & $7570-7782$ \\
One Nobel laureate in Chemistry & 34 & $8293-8667$ \\
Two Nobel laureates & 31 & $9292-9683$ \\
One Nobel laureate in Medicine & 25 & $11,536-11,981$ \\
One Nobel laureate in Chemistry & 18 & $15,780-16,696$ \\
One Nobel laureate in Medicine & 17 & $16,697-17,693$ \\
One Nobel laureate in Physics & 16 & $17,694-18,886$ \\
One Nobel laureate in Medicine & 15 & $18,887-20,273$ \\
One Nobel laureate in Physics & 13 & $21,761-23,427$ \\
One Nobel laureate in Chemistry & 12 & $23,428-25,533$ \\
One Nobel laureate in Physics & 10 & $27,952-31,043$ \\
\hline
\end{tabular}


scientists, who have certain number of highly cited papers. In particular, there are many thousands of individuals who have two-or-more-digit numbers of highly cited papers (much more than the number of HCR), and among them there are only 16 recent Nobel laureates (16\%, Economy not represented), and 36 recent Nobel laureates (37\%) have not published any highly cited paper while the number of scientists who have at least one highly cited paper substantially exceeds 100,000 . Table 5 suggests that the ability of publishing large numbers of highly cited papers is not common among recent Nobel laureates.

Among numerous bibliometric indicators presented by Ioannides et al. (2019) only a few were selected for the analysis of performance of recent Nobel laureates. There is no need to analyze them all because different bibliometric indicators based on citation records are highly correlated. These correlations were studied in detail by Ioannides et al. (2016), Schreiber et al. (2012), and many others. The following indicators were selected for further analysis:

- $c$ composite score (vide infra),

- $c$ (ns) composite score, self-citations excluded,

- Number of publications,

- Number of citations, self-citations excluded,

- ch, Hirsch index, self-citations excluded.

As discussed above, Ioannides et al. (2016) found only moderate correlation of their composite score with $h$, with the number of citations, and with the number of publications in top scientists, so we also do not expect high correlations in the ranks of recent Nobel laureates in these indicators. Table 6 shows top 10 ranks of recent Nobel laureates in the above

Table 6 Top 10 ranks of recent Nobel laureates in five bibliometric indicators, and the numbers of recent Nobel laureates in top 6000

\begin{tabular}{llllll}
\hline Rank in & $c(\mathrm{~ns})$ & $c$ & Papers & Citations $(\mathrm{ns})$ & $\mathrm{ch}$ \\
\hline & $19 \mathrm{~m}$ & $19 \mathrm{~m}$ & $308 \mathrm{~m}$ & $11 \mathrm{p}$ & $51 \mathrm{c}$ \\
& $73 \mathrm{p}$ & $83 \mathrm{p}$ & $563 \mathrm{c}$ & $13 \mathrm{p}$ & $91 \mathrm{~m}$ \\
& $93 \mathrm{~m}$ & $94 \mathrm{~m}$ & $976 \mathrm{c}$ & $201 \mathrm{~m}$ & $119 \mathrm{~m}$ \\
& $100 \mathrm{~m}$ & $98 \mathrm{~m}$ & $1214 \mathrm{c}$ & $204 \mathrm{c}$ & $182 \mathrm{~m}$ \\
& $116 \mathrm{c}$ & $112 \mathrm{c}$ & $1428 \mathrm{c}$ & $238 \mathrm{~m}$ & $255 \mathrm{c}$ \\
& $177 \mathrm{c}$ & $179 \mathrm{c}$ & $1543 \mathrm{p}$ & $256 \mathrm{c}$ & $300 \mathrm{c}$ \\
& $188 \mathrm{p}$ & $227 \mathrm{p}$ & $1620 \mathrm{p}$ & $356 \mathrm{~m}$ & $689 \mathrm{~m}$ \\
& $300 \mathrm{p}$ & $292 \mathrm{p}$ & $1838 \mathrm{p}$ & $392 \mathrm{c}$ & $689 \mathrm{p}$ \\
& $366 \mathrm{~m}$ & $355 \mathrm{c}$ & $2738 \mathrm{p}$ & $661 \mathrm{~m}$ & $733 \mathrm{p}$ \\
& $439 \mathrm{c}$ & $415 \mathrm{~m}$ & $3022 \mathrm{~m}$ & $992 \mathrm{~m}$ & $776 \mathrm{c}$ \\
\# in top 6000 & 45 & 45 & $11(+1)$ & $32(+2)$ & $32(+2)$ \\
Thereof: c & 18 & 18 & 4 & 11 & 13 \\
e & 4 & 4 & 0 & 1 & 0 \\
m & 16 & 16 & 3 & 13 & 14 \\
p & 7 & 7 & $4(+1)$ & $7(+2)$ & $5(+2)$ \\
Top-ranked e & 562 & 689 & 59,354 & 2493 & 11,452 \\
\hline
\end{tabular}

Numbers in parentheses: Nobel laureates not included in the compilation by Ioannides et al. (2019)

The abbreviations after the ranks: $c$ chemistry, $m$ medicine, $p$ physics 
five bibliometric indicators. The ranks are followed by the abbreviations of scientific disciplines represented by the Nobel laureates.

There are no recent Nobel laureates in top ten scientists in any of the five bibliometric indicators, and there are no recent Nobel laureates in 300 of the most productive scientists. Apparently the productivity is not the strongest side of recent Nobel laureates, and only 11 of them were in the top 6000 . The " $11+1$ " in the column "papers" in Table 6 denotes that on top of 11 highly productive recent Nobel laureates found in the compilation by Ioannides et al. (2019), there is another recent Nobel laureate who was not included in the compilation by Ioannides et al. (2019), but the manual search of Scopus indicated that he published more papers than the scientist ranked 6000 in that compilation. The fact that the productivity is not the parameter, in which the Nobel laureates stand out is well known, and the present results confirm that the famous statement of Garfield is still valid in spite of changing publication and citation practices. This is especially true for recent Nobel laureate in Economy who were all beyond the 59,000 most productive scientists.

There are two recent Nobel laureates in top 100 scientists in ch, and two recent Nobel laureates in top 100 scientists in the number of independent citations. Interestingly enough, they are four different individuals. Thirty-two $(+2)$ recent Nobel laureates (but no Nobel laureate in Economy) were in top 6000 scientists in ch, and thirty-two $(+2)$ recent Nobel laureates (including one Nobel laureate in Economy) were in top 6000 scientists in the number of independent citations. These numbers are much higher that the numbers of recent Nobel laureates among HRC, and in top 6000 scientists in hot papers, in highly cited papers and in productivity. The number of independent citations and ch are highly correlated, and there is a substantial overlap: $27(+2)$ recent Nobel laureates who are in top 6000 in ch are also in top 6000 scientists in the number of independent citations. Yet 58 recent Nobel laureates are neither in 6000 scientists in the number of independent citations nor in top 6000 scientists in ch.

The standings of recent Nobel laureates in the composite score $c$ are better than in any other bibliometric indicator discussed in this paper. There are four recent Nobel laureates in top 100 scientists in $c$, and 45 recent Nobel laureates in top 6000 scientists in $c$, and they are the same scientists in self-citation corrected and in uncorrected $c$. Table 6 shows a high correlation between the results obtained with and without self-citation correction. Such a correlation occurs only in top-cited scientists. Even in the compilation by Ioannides et al. (2019), which only covers relatively highly cited scientists, we can find substantial differences in the ranks of the same scientist between $c$ and $c$ (ns).

The present results qualitatively confirm the conclusion of Ioannides et al. (2016), but this study was performed with different set of data:

- Lifetime records rather than citations received within 1 year,

- Self-citation corrected rather than uncorrected citation records,

- Larger body of Nobel laureates (97 rather than 47 individuals).

\section{Conclusion}

Ninety-seven scientists received Nobel Prize in Chemistry, Economy, Medicine or Physics over the period 2010-2019. In order to assess the concurrence between the Nobel Prize and high standings in terms of several bibliometric indicators, the numbers of recent Nobel 
laureates in top 6000 scientists in terms of these indicators were compared. As many as 45 recent Nobel laureates were in top 6000 scientists in terms of $c$, a composite score introduced by Ioannides et al. (2016). No other indicator puts so many recent Nobel laureates in top 6000 as $c$. This result is remarkable because $c$ is not field-normalized, and the economists are at an inherent disadvantage. Nevertheless four recent Nobel laureates in Economy were in top 6000 scientists (from all disciplines) in terms of $c$.

Only 32 recent Nobel laureates were in top 6000 scientists in terms of ch (Hirsch-type index with self-citations excluded), and 32 recent Nobel laureates were in top 6000 scientists in terms of the number of citations (self-citations excluded). The number of citations and ch are also not field-normalized. The standings of recent Nobel laureates are even lower in field-normalized indicators. Only 17 recent Nobel laureates were among the 6000 Highly Cited Researchers (WoS®), 4 recent Nobel laureates were in top 6000 scientists in terms of the number of hot papers, and 2 recent Nobel laureates were in top 6000 scientists in terms of the number of highly cited papers.

Open Access This article is licensed under a Creative Commons Attribution 4.0 International License, which permits use, sharing, adaptation, distribution and reproduction in any medium or format, as long as you give appropriate credit to the original author(s) and the source, provide a link to the Creative Commons licence, and indicate if changes were made. The images or other third party material in this article are included in the article's Creative Commons licence, unless indicated otherwise in a credit line to the material. If material is not included in the article's Creative Commons licence and your intended use is not permitted by statutory regulation or exceeds the permitted use, you will need to obtain permission directly from the copyright holder. To view a copy of this licence, visit http://creativecommons.org/licenses/by/4.0/.

\section{References}

Biglu, M. H. (2008). The influence of references per paper in the SCI to Impact Factors and the Matthew Effect. Scientometrics, 74(3), 453-470.

Boettke, P. J., Fink, A., \& Smith, D. J. (2012). The impact of Nobel Prize winners in economics: Mainline vs. mainstream. American Journal of Economics and Sociology, 71, 1219-1249.

Dorta-González, P., \& Santana-Jiménez, Y. (2019). Characterizing the highly cited articles: A large-scale bibliometric analysis of the top $1 \%$ most cited research. Malaysian Journal of Library and Information Science, 24(2), 23-39.

Garfield, E. (1986). Do Nobel prize winners write citation classics? Essays of an Information Scientist, 9 , $182-187$.

Hirsch, J. E. (2005). An index to quantify an individual's scientific research output. Proceedings of the National Academy of Sciences of the United States of America, 102, 16569-16572.

Ioannidis, J. P. A., Baas, J., Klavans, R., \& Boyack, K. W. (2019). A standardized citation metrics author database annotated for scientific field. PLoS Biology, 17, e3000384.

Ioannidis, J. P. A., Klavans, R., \& Boyack, K. W. (2016). Multiple citation indicators and their composite across scientific disciplines. PLoS Biology, 14, e1002501.

Kosmulski, M. (2012). Calibration against a reference set: A quantitative approach to assessment of the methods of assessment of scientific output. Journal of Informetrics, 6, 451-456.

Kosmulski, M. (2018). Are you in top 1\% (1\%o). Scientometrics, 118, 557-565.

Kreiner, G. (2016). The Slavery of the $h$-index-Measuring the Unmeasurable. Frontiers Human Neuroscience, $10,556$.

Noorhidawati, A., Yanti Idata Aspura, M. K., Zahila, M. N., \& Abrizah, A. (2017). Characteristics of Malaysian highly cited papers. Malaysian Journal of Library and Information Science, 22(2), 85-99.

Sangwal, K. (2015). On the growth dynamics of citations of articles by some Nobel Prize winners. Journal of Informetrics, 9, 466-476.

Schreiber, M. (2009). A case study of the modified Hirsch index $h(m)$ accounting for multiple coauthors. Journal of the American Society for Information Science and Technology, 60, 1274.

Schreiber, M., Malesios, C. C., \& Psarakis, S. (2012). Exploratory factor analysis for the Hirsch index, 17 $h$-type variants, and some traditional bibliometric indicators. Journal of Informetrics, $6,347$. 